INDIRECT SUBJECTS 
This page intentionally left blank 
NOLLYWOOD'S

LOCAL ADDRESS

\section{Indirect Subjects}

Matthew H. Brown 
() 2021 Duke University Press

All rights reserved

Printed in the United States of America on

acid-free paper $\infty$

Project editor: Annie Lubinsky

Designed by Matt Avery

Typeset in MeropeBasic Regular by

Westchester Publishing Services

Library of Congress Cataloging-in-

Publication Data

Names: Brown, Matthew H., [date] author.

Title: Indirect subjects : Nollywood's local address / Matthew H. Brown.

Description: Durham : Duke University Press, 2021. | Includes bibliographical references and index.

Identifiers: LCCN 2020056100 (print) |

LCCN 2020056101 (ebook)

ISBN 9781478013280 (hardcover)

ISBN 9781478014195 (paperback)

ISBN 9781478021506 (ebook)

Subjects: LCSH: Motion pictures - NigeriaHistory. | Motion picture industry-

Nigeria-History. | Mass media and cultureNigeria-History. | Mass media-Political aspects-Nigeria-History. | Mass media policy-Nigeria. | BISAC: SOCIAL SCIENCE / Media Studies | PERFORMING ARTS / Film / History \& Criticism Classification: LCC PN1993.5.N55 B76 2021 (print) | LCC PN1993.5.N55 (ebook) | DDC 791.4309669-dc23

LC record available at https://lccn.loc .gov/2020056100

LC ebook record available at https://lccn.loc .gov/2020056101

Cover art: Stills from Checkmate (1991-1994, Nigeria). Crystal Gold/Moving Movies.

Duke University Press gratefully acknowledges the University of Wisconsin-Madison's Office of the Vice Chancellor for Research and Graduate Education, which provided funds toward the publication of this book. 